\title{
NORMAL MENTALITY ASSOCIATED WITH A MALDEVELOPED "RHINENCEPHALON"
}

\author{
BY \\ P. W. NATHAN and MARION C. SMITH \\ From the Neurological Research Unit of the Medical Research Council, National Hospital, \\ Queen Square, London
}

In 1937 Papez published his views on the functions of the gyrus fornicatus. He summarized them as follows.

"It is proposed that the hypothalamus, the anterior thalamic nuclei, the gyrus cinguli, the hippocampus, and their inter-connexions constitute a harmonious mechanism which may elaborate the functions of central emotion, as well as participate in emotional expression."

Bilateral temporal lobectomies performed on monkeys by Klüver and Bucy in 1939 gave evidence supporting Papez' general hypothesis. The effects of extensive lesions of the hippocampus-fornix system in cats and dogs were reported by Spiegel, Miller, and Oppenheimer (1940) ; restricting the extent of the lesions, they found that "definite outbursts of rage were observed after bilateral destructions of the amygdaloid nuclei". Further experimental investigations on the gyrus fornicatus and its connexions were carried out by Bard and Mountcastle (1948). They found that "sham rage", which usually occurs in cats as a result of the removal of the cerebral cortex, did not occur if the rhinencephalon and the gyrus cinguli were left intact; when these structures, or the gyrus cinguli alone, were removed later, typical "sham rage" appeared. When both hippocampi were removed from cats " an exaggeration of pleasure reactions " appeared, and cats which were unfriendly before operation became friendly and demonstrative. Gerebtzoff (1941) investigated the distribution of the fornix in the guinea-pig and rabbit. Allen (1944) traced the fibres of the fornix by means of the Marchi method in dogs. Brodal (1947), and Le Gros Clark and Meyer (1947) delimited the parts of the brain which receive olfactory connexions from the rest of the gyrus fornicatus. As the abnormality in the specimen to be described is within the parts of the telencephalon which have been the object of these investigations, it seemed desirable that its features should be recorded.

D

\section{Case Report}

The specimen is the brain of a man, aged 34 , who died of a chondrosarcoma of the ileum. He was one of a series of cases under investigation in a programme of research concerned with operations for the relief of pain. As many hours had been devoted to the testing of sensation and the discussion of signs and symptoms, our knowledge of the patient, which extended over a period of six months, was much greater than that acquired in most routine cases.

No abnormality of development had been suspected during the patient's life, for he came well within normal limits from intellectual and psychological points of view. His mother had remained well during pregnancy, and his birth was normal. There were no external congenital abnormalities and no clinical evidence of abnormality in his nervous system was found.

From our knowledge of the patient, we would consider " easy-going" as an apt description of his personality ; his mother described him as lazy. He was always sociable and very popular, ready to joke, and of a sympathetic nature. He was obviously intelligent; his mother said : "He was as sharp as a needle, but a bit lazy". He had left school in the top class, although he did less well than his siblings. In reply to questioning, his mother said he did not have a temper. "He wouldn't bother to lose his temper"; he was never angry, and never grumbled. What he enjoyed most was betting at greyhound races; and he would spend much of his time with people connected with the race-track. He also liked painting, drawing, and making rugs. His work before the war, which was window-dressing, was on the same social and financial level as that of his father and siblings. He served in the Army for five years of the recent war, remaining a private soldier throughout. $\mathrm{He}$ remained unmarried, although there had been one or two friendships with girls.

It is to be noted that he possessed a normal sense of smell. No congenital abnormality was found post mortem in any part of the body except the cerebral hemispheres.

\section{Methods}

Within three hours of death, $10 \%$ formol saline was injected into the cisterna magna; the brain and spinal 
cord were removed within 24 hours of death and fixed in $20 \%$ formol saline for three weeks. The brain was then divided in the median sagittal plane and the left hemisphere sliced coronally into blocks $\frac{1}{2} \mathrm{~cm}$. thick. A thin block of tissue including the entire length of the corpus callosum and overlying grey matier was taken from the right hemisphere. All blocks were embedded in celloidin, and sections were stained by the following methods: Ehrlich's hæmatoxylin and eosin, thionin, Mallory's phosphotungstic acid hæmatoxylin, Loyez' and Gros' stains. In all important areas serial sections were made. Similar blocks and sections were made of normal brains for comparison.

\section{Macroscopic Examination}

The uncut brain showed no striking abnormality on its dorsal and lateral surfaces, but when the hemispheres were drawn apart, instead of the corpus callosum, gyri were seen passing across the median plane connecting the two hemispheres. It was necessary to cut through these transversely running gyri in order to separate the hemispheres; it was then seen that the corpus callosum and gyrus cinguli were replaced by an irregular mass of white matter covered by overlying grey matter (Fig. 1). There is no trace of a continuous

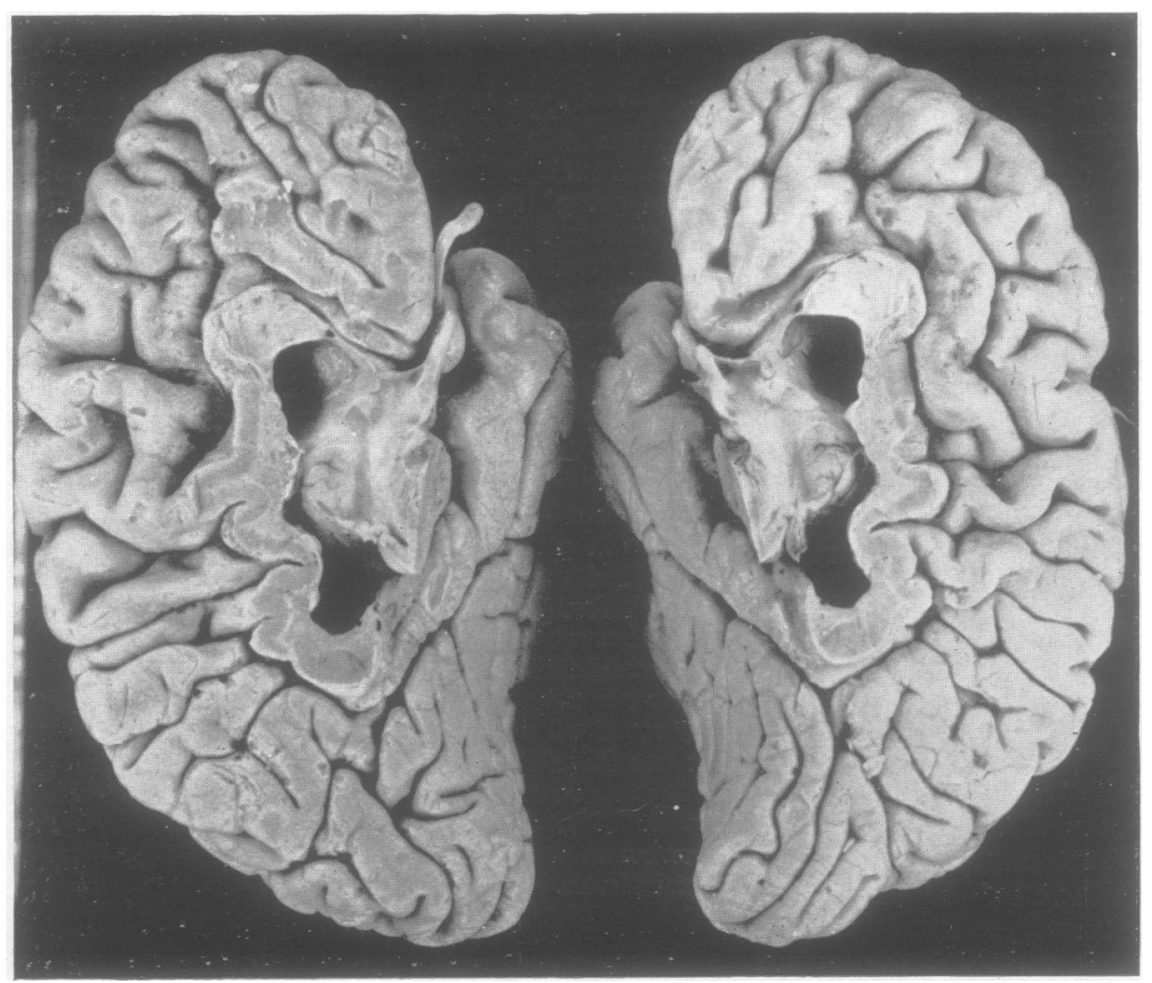

FIG. 1.-Medial view of cerebral hemispheres. longitudinal cingulate gyrus or sulcus. The mass of white matter joining the two hemispheres possesses anterior and posterior enlargements comparable in size and position to the normal genu and splenium (Fig. 2). On its ventral surface are several small masses of ectopic grey matter; these are numerous in the roof of the lateral ventricles, where they partly obliterate the cavity. The corpus callosum is partly adherent to the thalamus and to the caudate nucleus. The hippocampus is joined directly to the splenium of the corpus callosum and to its fellow of the opposite side, thus forming a transverse vault in the complete specimen. The white matter connecting the two hemispheres will be referred to as the corpus callosum.

The isthmus of the gyrus fornicatus is represented by a broad posterior continuation of the hippocampal and dentate gyri. This displaces the lingual gyrus medially, altering its relations and configuration, so that it comes to form part of the medial surface of the hemisphere.

As the most striking feature of this brain is the absence of the fimbria and fornix, the report of the further examination of it will be confined for the most part to the structures related to the fornix system, namely the hippocampal formation (hippocampus or cornu Ammonis, hippocampal gyrus, dentate gyrus), fimbria, septum pellucidum, hypothalamus and mamillary bodies, amygdaloid complex, and corpus callosum.

In addition to the absence of the fornix, there is no septum pellucidum ; the $a b-$ sence of these structures results in such wide continuity between the cavities of the third and lateral ventricles, that there is in effect one large cavity.

The hippocampus and the dentate gyrus are displaced caudally ; they lie entirely posterior to the 


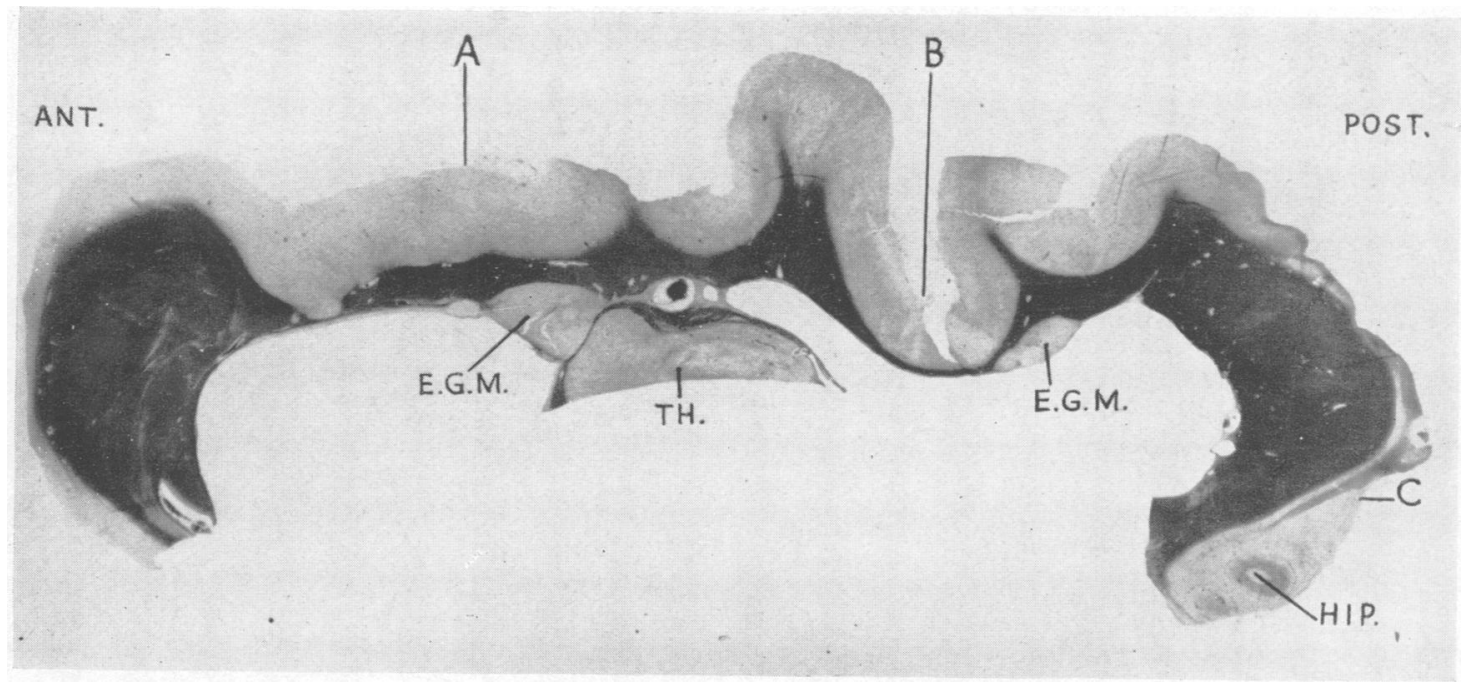

FIG. 2.-Sagittal section through corpus callosum and overlying grey matter (Loyez' myelin stain). A, B, and C refer to divisions into types of cortex, described in the text.

amygdaloid complex, which is nevertheless more extensive than in the normal brain (Fig. 3). Posteriorly the hippocampus and dentate gyrus are continuous with their fellows of the opposite side. They are poorly developed in all dimensions, but less so in the posterior third. The hippocampal gyrus is also grossly under-developed throughout its entire length; its small size can be seen in Fig. $4 b$; at this level it should be much bigger than the hippocampus and dentate gyrus, as is seen in Fig. 4a. The hippocampal fissure is absent. In association with the small size of the hippocampal gyrus there is a great increase in depth of the collateral sulcus with its surrounding grey matter, so that the major part of the floor of the ventricle is formed by an enormous collateral eminence, and the hippocampus and dentate gyrus are displaced medially and ventrally (Figs. 5 and 6 ). Owing to this displacement and the small size of the hippocampal gyrus the hippocampus and dentate gyrus can be seen from the ventral surface of the brain, instead of lying within the ventricle hidden by the hippocampal gyrus as in the normal. These features and the entire absence of the hippocampal fissure and the great reduction in number of the denticulate convolutions combine to give the grossly abnormal appearances seen in the transverse sections.

The olfactory tract occupies its normal position. There is no massa intermedia. The anterior, posterior, and habenular commissures are normal.

\section{Microscopic Examination}

Although the fornix itself is absent, alveus fibres are present, but they are less numerous than in the normal brain. A very few fibres can be traced from the alveus to the position of the fimbria, but they are so few that they do not form a macroscopic structure. The position of the fimbria is occupied by a mass of grey matter which joins the dentate gyrus to the retrolenticular part of the internal capsule and to the medial aspect of the tail of the caudate nucleus. The fibres of the hippocampal commissure show no reduction in number. Throughout the extent of the hippocampal gyrus fibres are seen running at right angles to the cortex; they are presumably the fibres of the cingulum.

The extent and appearance of the nuclear groups which normally receive most of the fibres of the fornix are within normal limits. The corpora mamillaria lack their main afferent pathway, for the anterior pillars of the fornix are absent; but their efferent tracts, the mamillo-thalamic and mamillo-tegmental, appear to be completely normal. The shape of these bodies is within normal limits and all mamillary nuclei are present. Cell counts show that the medial, intercalatus, and the supramamillary nuclei have a normal cell population; the lateral nucleus is not quite as clearly demarcated as in some of the control specimens, but appears to be within normal limits.

The fornix is said to carry fibres also to the anterior part of the hypothalamus, the septal nuclei and the habenula. The hypothalamus was examined in serial sections throughout this region. All nuclei are normal, with the following minor exceptions. The tuber nuclei are in the usual definite groups, but contain fewer cells than normal. 

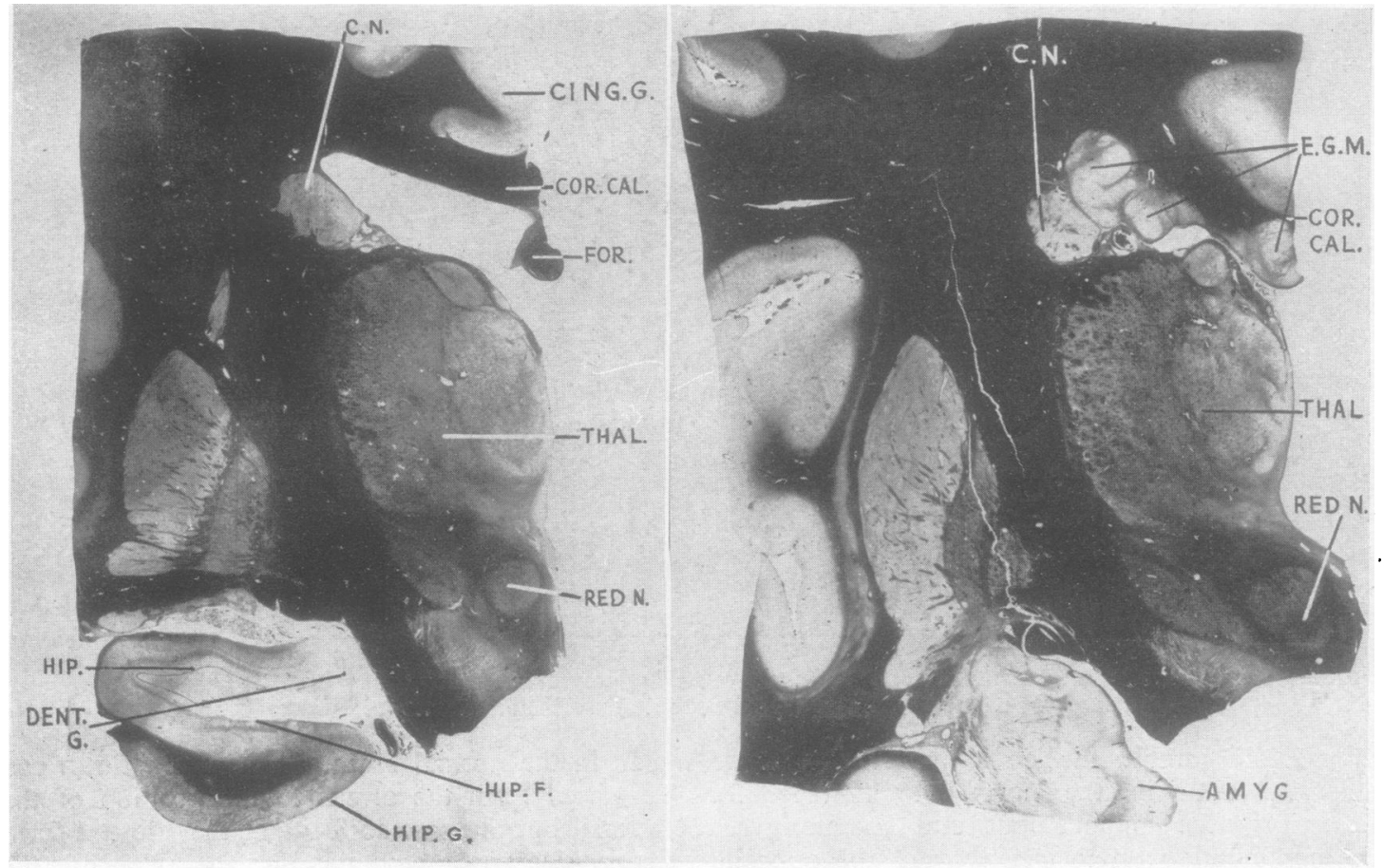

Fig. 3.-Coronal sections ( $a$, normal ; $b$, abnormal) of left hemisphere at level of the red nucleus (Loyez' myelin stain).
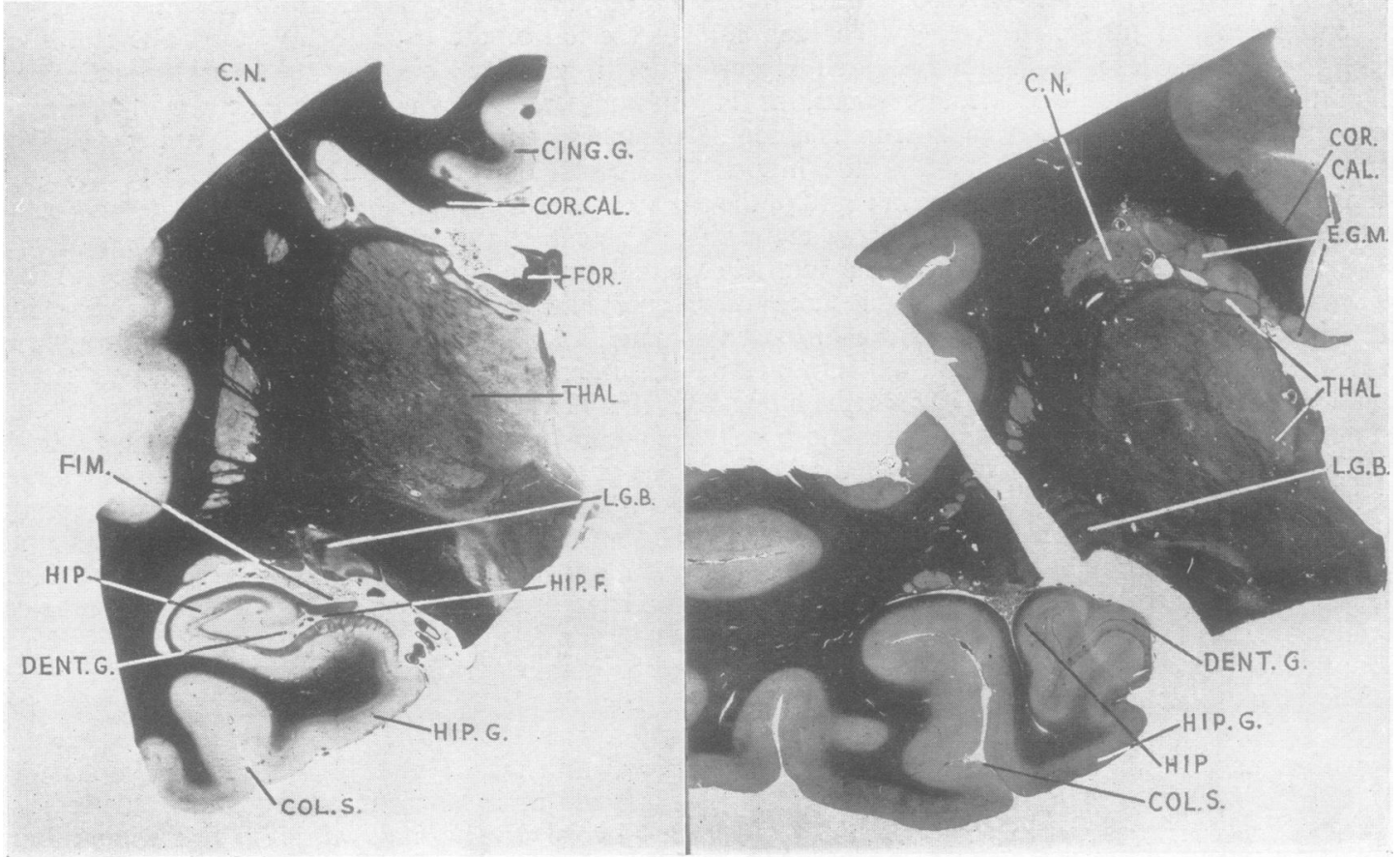

FIG. 4.-Coronal sections ( $a$, normal ; $b$, abnormal) of left hemisphere at level of lateral geniculate body (Loyez' myelin). 

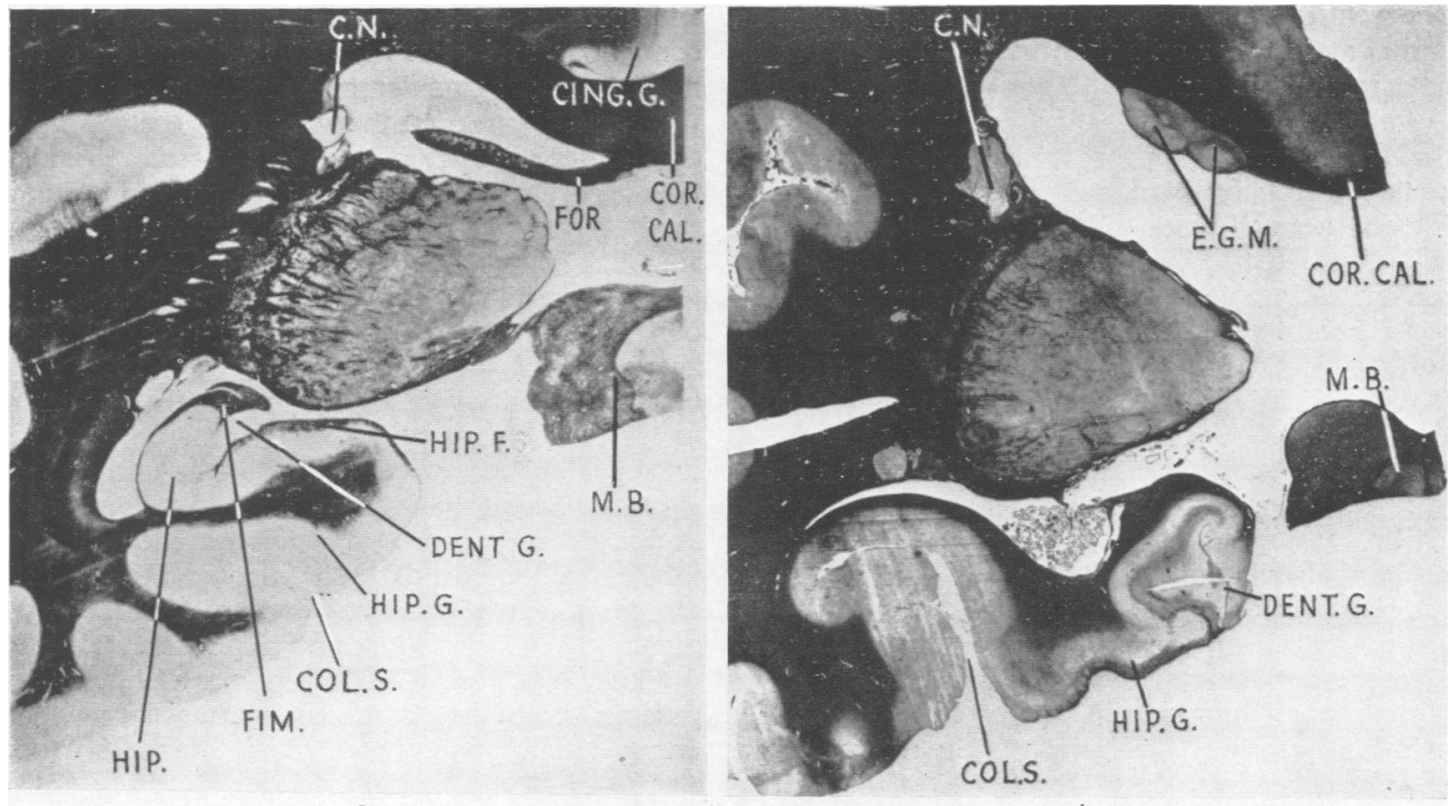

FIG. 5.-Coronal sections ( $a$, normal ; $b$, abnormal) of left hemisphere at level of pulvinar (Loyez' myelin stain).
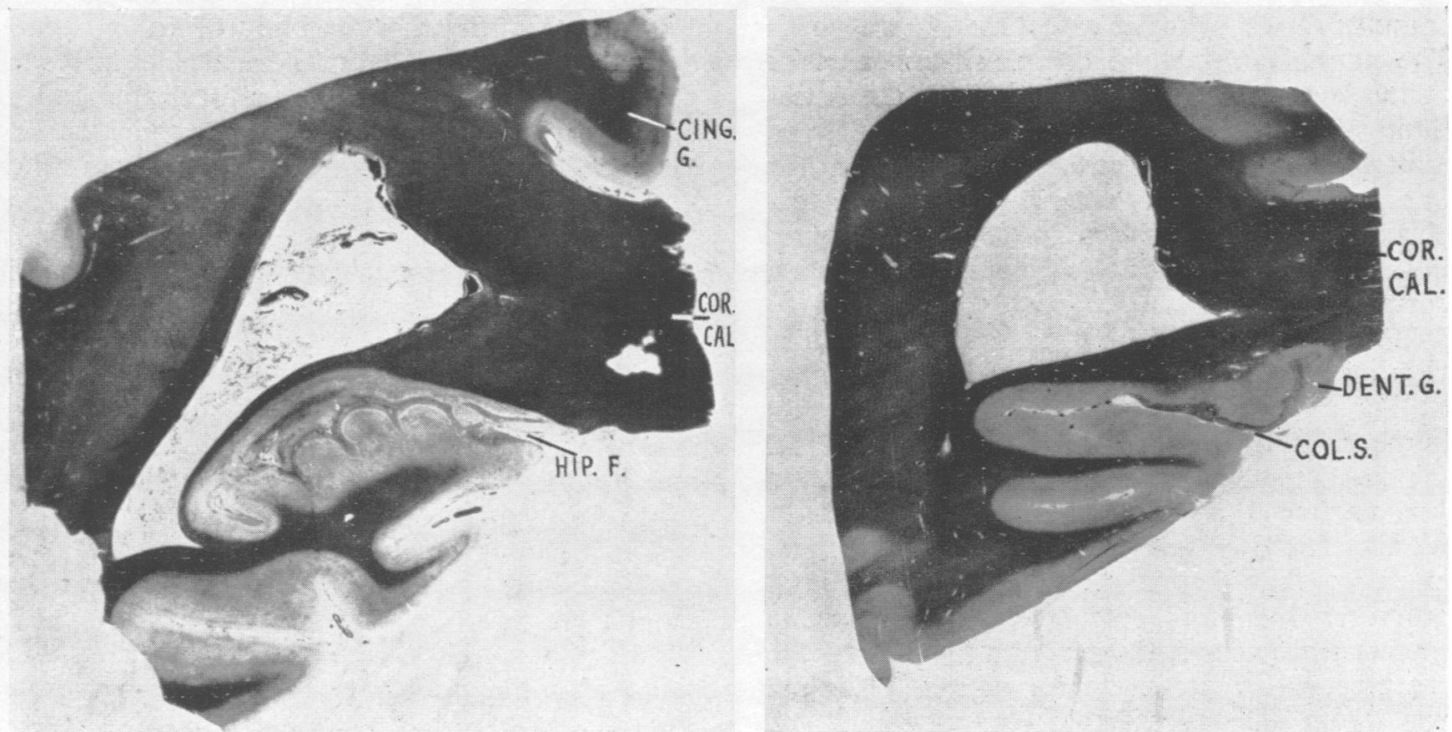

Fis. 6.-Coronal sections ( $a$, normal ; $b$, abnormal) of left hemisphere at level of splenium of corpus callosum (Loyez' myelin stain).

Ant. : anterior. post. : posterior. amyg. : amygdaloid. c.n. : caudate nucleus. cing. g. : cingulate gyrus. col. s. : collateral sulcus. cor. cal. : corpus callosum. dent. g. : dentate gyrus. e.g.m. : ectopic grey matter. fim. : fimbria. for. : fornix. hip. : hippocampus. hip. g. : hippocampal gyrus. hip. f. : hippocampal fissure. l.g.b. : lateral geniculate body. m.b.: mid-brain. red n.: red nucleus. th.: thalamus. 
The ventromedial nucleus may be slightly smaller and contain fewer cells than in the normal, but is not grossly deficient.

Although this brain has no septum pellucidum, the septal nuclei appear to be present. In the sections through the rostral part of the hemisphere there is a small mass of grey matter lying medial and dorsal to the nucleus accumbens septi whose cell characteristics suggest that this grey matter consists of the nuclei which are normally located in the septum. The nucleus accumbens septi is in its normal position and of normal shape and size.

The habenular nuclei are within the limits of the normal.

The cytological features of the anterior nucleus of the thalamus and of all thalamic nuclei, of the caudate nucleus, putamen, and the globus pallidus are normal throughout.

In spite of the macroscopic abnormalities of the hippocampal complex, the component cells of each of its parts are normal ; obviously the total number of cells is reduced since the structures themselves are so small. The characteristic pyramidal and granular cells can be recognized in the midline beneath the splenium, where the hippocampal complexes of the two sides join. The amygdaloid complex is of far greater extent than in the normal. The normal amygdaloid complex starts anteriorly at the level of the optic chiasma and the anterior limit of the globus pallidus; the mass of its grey matter ends posteriorly at the level of the mamillary bodies. Here it starts anteriorly behind the optic chiasma, that is about $1 \mathrm{~cm}$. posterior to its normal position; it ends posteriorly at the level of the corpus subthalamicum, about $3 \mathrm{~cm}$. posterior to its normal position. This increased size is associated with an increased spacing out of its cells, without, as far as can be judged, any increase in their number. Although cells having the characteristics of the amygdaloid nuclei can be identified, the various nuclei are not as clearly demarcated as usual. The lateral nucleus in particular is far more extensive than in the normal; all other nuclei are somewhat more spread out than normally; the cortical nucleus, the peri-amygdaloid area, and the prepyriform cortex appear normal. The pathway from the amygdaloid complex, the stria terminalis, is normal.

The main bulk of the white matter forming the corpus callosum is composed of transversely and obliquely running fibres, which are most numerous at the anterior and posterior extremities; a thin band of longitudinal fibres forms the external layer. The grey matter covering the corpus callosum varies greatly in thickness; its cyto- architectonic structure is the same as that of the contiguous gyri above it. It is divided into two main types; agranular and granular. In Fig. 2, the point $B$ marks the boundary between the anterior agranular and the posterior granular type of cortex. This distribution of cytoarchitectonic types corresponds with that of the normal gyrus cinguli. Between the points $A$ and $B$, the cortex, though agranular in type, differs from that of the normal gyrus cinguli in that the pyramidal cell layers are thicker, and very large pyramidal cells, having all the characteristics of Betz cells, are found in the deepest layer. Posterior to the point $\mathrm{C}$, the cortex is typical of the hippocampus and dentate gyrus. The ectopic masses lying on the ventral aspect of the corpus callosum are divided into lobules by thin bands of myelinated fibres; they are composed of medium- and small-sized nervecells and of all three types of glia, astrocytes being the least common. The appearance is not homogeneous, as the cells are to some extent layered and grouped, although irregularly; this formation suggests a cortical origin. They are quite unlike the adjacent caudate nucleus or thalamus.

\section{Discussion}

It is noteworthy that the brain of an apparently normal man who lived a normal life has such gross abnormalities in the gyrus fornicatus and its connexions. These abnormalities include an abnormal and very small hippocampus, from which no efferent pathway has been found, except one to its fellow of the opposite side, and an abnormal cingulate gyrus and corpus callosum. As the fornix and the hippocampal commissure are the only known corticofugal pathways from the hippocampus, it is difficult to see how this part of the brain functioned. This absence of fornix is associated with normally developed corpora mamillaria; the alveus, of which the normal fornix is a continuation, is fairly well developed. If " the hippocampus must be considered as an effector structure" (Brodal, 1947), this brain has an effector structure with no effector path, except for a commissural one to its fellow of the opposite side ; also, the groups of cells into which its fibres should lead are within normal limits. As might be expected on the basis of recent anatomical research, this man had no abnormalities in his sense of smell. He had no lack of emotional control and was not prone to rages, he showed no psychiatric symptoms, and no difficulty in dealing with or expressing emotion. It is true that congenital abnormalities may throw little light on function, for one is always impressed by the manner in which 
normal function is attained in spite of apparently inadequate structure.

In a search through the literature of congenitally abnormal brains, only one was found resembling this one (Fleming and Norman, 1942). In their specimen there was interdigitation of gyri which " are apparently derived from the cingulate gyrus ... Further back, between the parietal lobes, grey matter, not a corpus callosum, connected the two halves of the brain. This smooth plate of tissue was continuous laterally with the cingulate gyrus, and ended posteriorly in a bulbous extremity above the tegmental part of the midbrain. This rounded termination resembled in longitudinal section the splenium of a corpus callosum, and was structurally continuous on each side with the posterior ends of the hippocampal gyri. . . . Two thin strands of white matter ... entered the hippocampus of each side as the posterior pillars of the fornix". From their photographs it is seen that the corpus callosum was much thicker than that of the brain described here; it is also probable that the hippocampal gyri were smaller than normal.

The many brains recorded with no corpus callosum are quite unlike the brain described here.
Summary

A description and photographs of a brain are given, in which bilateral abnormalities of the hippocampus and absence of the fornix are associated with normal olfactory function and normal emotional control.

We take this opportunity of thanking Mrs. M. Meyer for her interest and advice, Professor F. Goldby for his help in writing this paper, and Miss Kathleen Harrison and Miss Vera Burgess for their invaluable technical assistance.

\section{REFERENCES}

Allen, W. F. (1944). J. comp Neurol., 80, 283.

Bard, P., and Mountcastle, V. B. (1948). Res. Publ. Ass. nerv. ment. Dis., 27, 362.

Brodal, A. (1947). Brain, 70, 179.

Clark, W. E. Le Gros, Beattie, J., Riddoch, G., and Dott, N. M. (1938). "The Hypothalamus." Edinburgh.

- - and Meyer, M. (1947). Brain, 70, 304.

Fleming, G. W. T. H., and Norman, R. M. (1942) J. ment. Sci., 88, 341.

Gerebtzoff, M. A. (1941). J. belge Neurol. Psychiat., 41, 199.

Klüver, H., and Bucy, P. C. (1939). Arch. Neurol. Psychiat., Chicago, 42, 979.

Papez, J. W. (1937). Ibid., 38, 725.

Spiegel, E. A., Miller, H. R., and Oppenheimer, M. J. (1940). Trans. Amer. neurol. Ass., 66, 127.

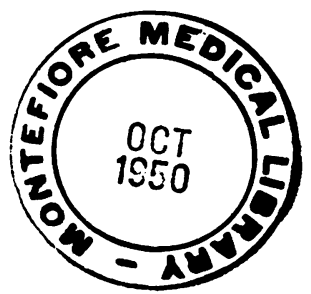

